\title{
Improvement of cosmic-ray muography for Earth sciences and civil engineering
}

\author{
László Oláh* \\ Earthquake Research Institute, University of Tokyo \\ E-mail: olah.laszlo@wigner.mta.hu, olah@eri.u-tokyo.ac.jp
}

Hiroyuki K. M. Tanaka

Earthquake Research Institute, University of Tokyo

E-mail: ht@muographix.u-tokyo.ac.jp, ht@eri.u-tokyo.ac.jp

\section{Gergő Hamar}

Wigner Research Centre for Physics of the Hungarian Academy of Sciences

E-mail: hamar.gergo@wigner.mta.hu

\section{Dezső Varga}

Wigner Research Centre for Physics of the Hungarian Academy of Sciences

E-mail: varga.dezso@wigner.mta.hu

\begin{abstract}
Muography is utilizing the cosmic-ray muons to deduce the amount of material across large-sized objects, similarly to X-raying of human body. We specifically developed Multi-Wire Proportional Chamber (MWPC)-based tracking systems with high ( $>98 \%)$ efficiency, fair $(<10 \mathrm{mrad})$ angular resolution and low $(<6 \mathrm{~W})$ power for Earth sciences and civil engineering. A muography observatory is assembled from trackers on a sensitive surface of $5.5 \mathrm{~m}^{2}$ at $2.8 \mathrm{~km}$ distance in South-West from Sakurajima volcano to provide projective density images of the crater regions for future measurements of mass variations occurred during eruptions. An industrial applicability of portable, MWPC-based instruments have been demonstrated with the muographic imaging of an underground iron pillar.

We investigated the limits of muography: the Gaisser model is suggested to be modified with an energy exponent of -2.64 and constant scale factor of 0.66 for imaging in near-horizontal directions after large (>1,000 m.s.r.e.) thicknesses. The multiple scattering of muons across the targeted object is limiting the imaging resolution from $10 \mathrm{mrad}$ to $5 \mathrm{mrad}$ with the increase of thickness between 50 m.s.r.e. and 2,000 m.s.r.e..

The precise measurement of low-energy muon spectra is required to improve muography of smallsized objects. We developed a 5-meter-length, rotatable, MWPC-based spectrometer to precisely measure the energy spectra of muons between $0.5 \mathrm{GeV}$ and $5 \mathrm{GeV}$ from vertical to horizontal directions. It is a consecutive series of thirteen detectors with a positional resolution of approx. 4 $\mathrm{mm}$ and lead plates. The spectrometer and the first results are presented.
\end{abstract}

36th International Cosmic Ray Conference -ICRC2019-

July 24th - August 1st, 2019

Madison, WI, U.S.A.

${ }^{*}$ Speaker. 


\section{Introduction}

Muography is an imaging technique that derives the amount of material across large-sized objects by the measurement of the flux of cosmic-ray muons [1], similarly to the X-raying of human sized objects. Particle physics innovations has allowed the adaptation of muon trackers to outdoor conditions that led to the first successful applications, e.g. the density structure has been captured [2], and later density variations were being monitored [3] across erupting volcanoes by cosmic muon tracking. Despite of the pioneering works, the application oriented development of instrumentation and improvement of imaging techniques are required to make muography a standard imaging technique. In this paper, we will review our hardware developments and cosmic-ray physics related studies performed to provide high-quality muography data for various applications.

\section{A modular muography observatory for density monitoring of volcanoes}

Muography can derive the amount of material across volcanoes with a spatial resolution of a few tens of meters that can not be achieved by conventional observation techniques [2]. Thus, the permanent observation of mass movements inside active volcanoes would provide useful auxiliary information to both volcanology and disaster research [3]. The imaging of km-sized bodies requires the application of a Muography Observation System (MOS) with large surface area and high angular resolution. This detector arrangement can measure desirable number of muon tracks, provide reasonable spatial resolution from a safe distance and suppress the background.
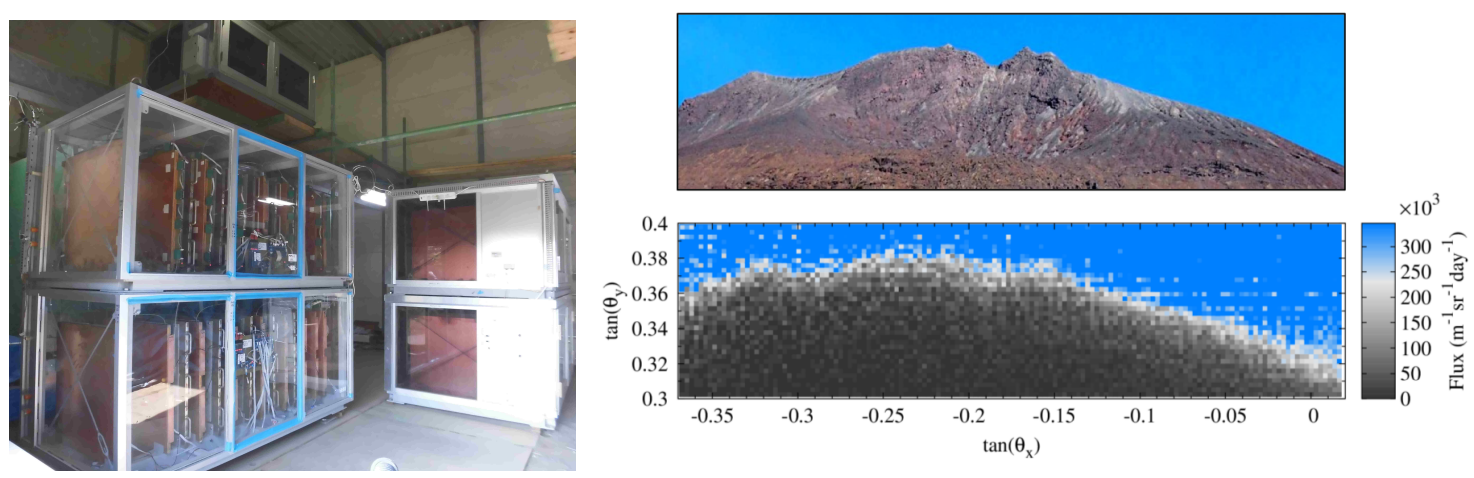

Figure 1: A photograph of Sakurajima Muography Observatory (SMO, left). A photograph (upper right) and the muon flux (lower right) measured across Minamidake are shown from a distance of $2.8 \mathrm{~km}$ [4].

A Multi-Wire Proportional Chamber (MWPC)-based MOS (MMOS) [4, 5, 6, 7] is under development at Sakurajima volcano that is one of the most active Japanese volcanoes with hundreds of explosive eruptions per year. The MMOS is a modular observatory assembled from 2-meterlength tracking systems (Figure 1a). Each module has at least five MWPCs and five 2-cm-thick lead plates. These MWPCs provide a positional resolution of approx. $3.5 \mathrm{~mm}[8]$ and an angular resolution of about $3 \mathrm{mrad}$ that is equivalent with a spatial resolution of approx. $10 \mathrm{~m}$ across the craters located at a distance of $2.8 \mathrm{~km}$ from the MMOS [4]. Figure $1 \mathrm{~b}$ shows the measured flux that resolves the peaks of Sakurajima located at a distance of a few tens of meters on the crater rim and demonstrate the applicability of MMOS for high-definition muon imaging [4]. 
Actually, the MMOS consists of seven tracking systems and covers a sensitive surface of $5.5 \mathrm{~m}^{2}$. Local electricity network and surge protection provides its continuous operation. The data acquisition is controlled by a microcomputers on each tracker, and the trackers are controlled by a server micro-computer. The total power consumption of an MMOS is approx. $6 \mathrm{~W}$. The trigger is given by a triple coincidence of MWPCs. The data files are written on event-by-event with the track positions on each MWPC, the trigger pattern with its timing, and the analogue signal amplitudes as ASCII files. The data are transferred to a remote computer where the data reconstruction, quality assurance are performed to provide data visualization via an online-user interface [7,9]. The tracking systems measure the charge particles with a typical rate of 0.07$0.2 \mathrm{~Hz}$ with a typical variation of below $5 \%$ [7]. Therefore, the MMOS is expected to observ the typical flux variations of 5-15\% caused by the density-length variations of 5-10\% occurred before/after volcano eruptions [3].
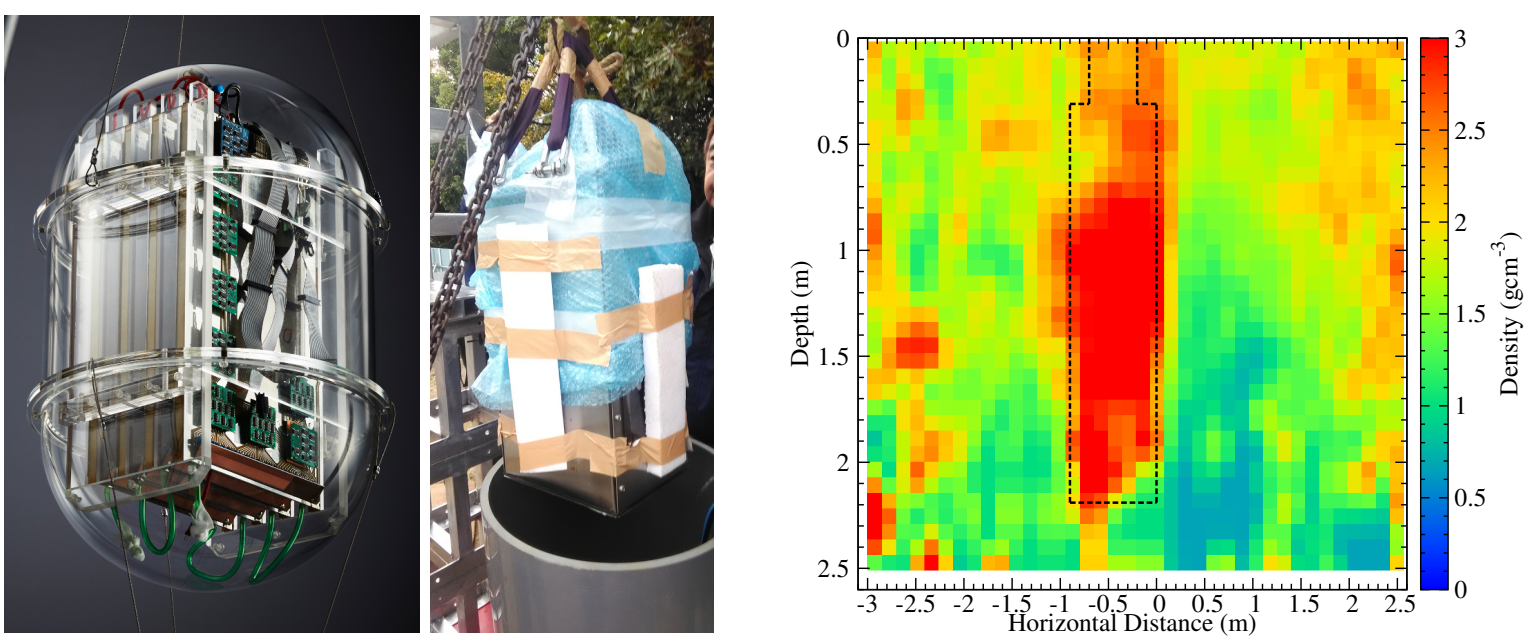

Figure 2: A photograph about portable muography instrument (left), about its installation in a shaft (middle), and a muographic image (right) of a concrete pillar [10], where dashed line shows its expected position.

\section{A portable muographic observation instrument for civil engineering}

For inspection of the structure of human-made constructions, such as concrete pillars, bridges, dams, or buildings, we developed a compact, portable, low-power $(5 \mathrm{~W})$ instrument with high (>98\%) detection efficiency [10]. This instrument is based on close-cathode chamber technology [11] and the so called Muontomograph that is applied for exploration of natural caves [12]. We cooperated with NEC corporation to validate the applicability of the portable MMOS for civil engineering. NEC prepared an experimental site on a 3-meter-high mound with a $50 \mathrm{~cm}$-radii shaft where the MMOS was installed. As an observation target, a standard pillar of Japanese Railway was installed at approx. $3 \mathrm{~m}$ distance from the MMOS. Aim was to measure the bottom of the pillar with a precision of approx. $10 \mathrm{~cm}$ to demonstrate the applicability of muography for detection of possible structural damages in pillar structures. Figure 2 shows the photograph of a portable instrument (left), the installation of an MMOS inside the shaft (middle), and the muographic image 
of the pillar (right) taken during 14 days. This image revealed successfully the bottom of the pillar at approx. $80 \mathrm{~cm}$ above the detector level. Although, the MMOS demonstrated its applicability in an industrial environment, such as in boreholes, the development of gas supplying will be required for its practical application by non-experts.

\section{Limits and feasibilities of high-definition muon imaging}

Muography is based on the accurate modeling of the measurements to extract interpretable physical quantities across the objects, such as average density across the volcanoes. At each path across the object, the modeled flux is simulated or calculated by an integration of muon spectrum from a minimum energy that is required for muons to penetrate the object. The Modified-Gaisser model [13] can parametrize the muon spectra in the complete angular and energy ranges. For volcano muography the spectra has to be fitted more precisely at larger zenith-angles $\left(\vartheta_{y}>60^{\circ}\right)$. For this aim, we adjusted the energy exponent $(\gamma)$ and constant factor $(C)$ parameters of the ModifiedGaisser model, and compared the calculated fluxes to the existing experimental data collected at large depths [5]. Figure 2 shows that the fluxes calculated with $\gamma=-2.64$ and $C=0.66$ (solid lines) fit more precisely on the experimental data $[14,15,16]$ (open dots) than the ones with the original parameters (dashed lines). The relative flux differences $\left(\left(F_{\text {measured }}-F_{\text {expected }}\right) / F_{\text {measured }}\right)$ were found to be $10.4 \%$ for the original- and $1.6 \%$ for the suggested parametrization, respectively.

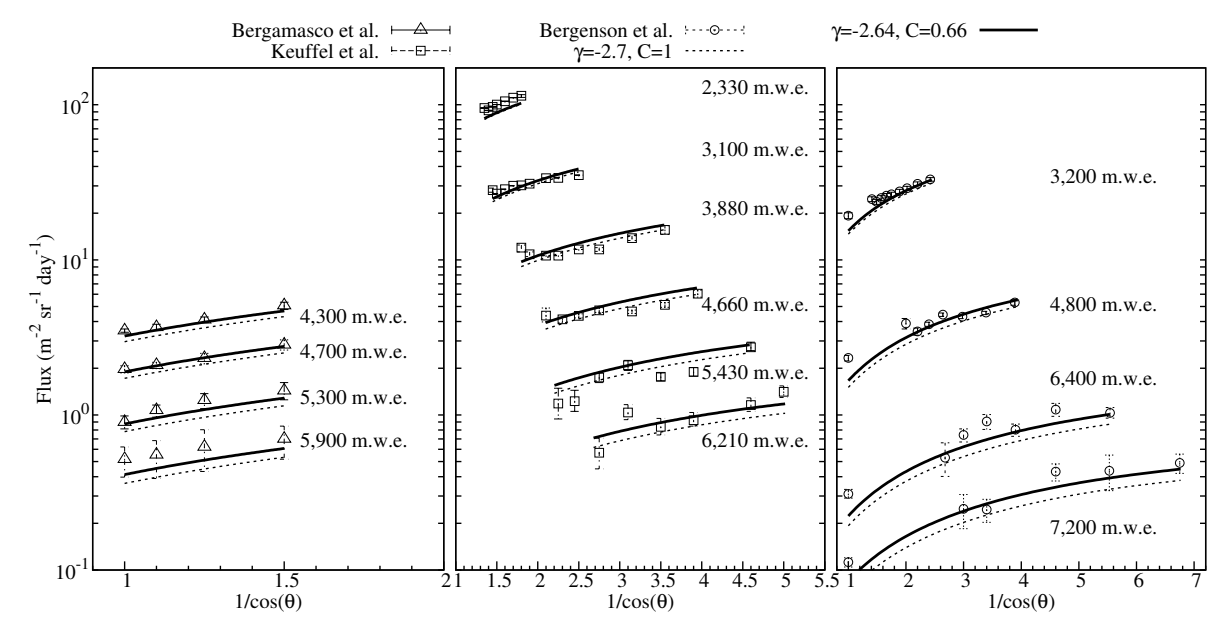

Figure 3: The comparison of Modified Gaisser model with the original (dashed lines) and suggested (solid lines) parameters to the experimental data (open dots) collected at large depth under large zenith-angles [5].

Muographic observation has to find a compromise between the angular resolution of imaging $(\Delta \alpha)$ and the measurement time $(\Delta T)$. In case of volcanoes the $\Delta \alpha$ has to be enough high to resolve crater structure that is approx. $100 \mathrm{~m}$, and the measurement time has to be quite short to observe the occurred volcanological processes that takes from few hours to few weeks. Another constrain on $\Delta \alpha$ is given by the multiple scattering of muons: $\Delta \alpha \geq \sqrt{\sigma_{\text {Obj+Air }}^{2}+\sigma_{\text {MOS }}^{2}}$, where $\sigma_{\text {Obj+Air }}$ and $\sigma_{\mathrm{MOS}}$ are the multiple scattering of muons until the tracker, and across the tracker. To quantify the effect of multiple scattering [5], various sized objects and an MOS were implemented in standard atmosphere with different distances, and the penetration of muons were simulated from 
the object ot the MOS in GEANT4 framework [17]. The expected scattering angles of muons $\left(\Delta \theta_{\mathrm{Obj}+\mathrm{Air}}\right)$ after their penetration across the object and air $\left(d_{\mathrm{Obj}}+\mathrm{Air}\right)$ were calculated from the simulated data sets as follows.

$$
\Delta \theta_{\mathrm{Obj}+\mathrm{Air}}\left(d_{\mathrm{Obj}+\mathrm{Air}}\right)=\frac{p_{v}\left(d_{\mathrm{Obj}+\mathrm{Air}}\right)}{p_{h}\left(d_{\mathrm{Obj}+\mathrm{Air}}\right)}-\frac{x_{v}\left(d_{\mathrm{Obj}+\mathrm{Air}}\right)-x_{v}\left(d_{0}\right)}{x_{h}\left(d_{\mathrm{Obj}+\mathrm{Air}}\right)-x_{h}\left(d_{0}\right)},
$$

where $p_{v}$ and $x_{v}$ describe the vertical momentum directions and coordinates, $p_{h}$ and $x_{h}$ express the corresponding horizontal ones, and $\mathrm{d}_{0}$ is $x_{h}(0)$, i.e. particle position at the further side of the object. The distribution of these scattering angles were determined and the multiple scattering $\left(\sigma_{O b j+A i r}\right)$ values were quantified by calculating the root-mean-square values for the central $98 \%$ of the data set. Figure $\mathrm{X}$ shows the zenith-angle dependent ratio of momentum directions and multiple scattering as a function of distance from the object for 100, 500, 1000, and 2000 meterstandard-rock-equivalent (m.s.r.e) objects, respectively. The multiple scattering decreases from $15 \mathrm{mrad}$ to $5 \mathrm{mrad}$ with the increase of thickness due to the hardening of the muon spectra at larger energies that required to penetrate larger thicknesses. Furthermore, the multiple scattering decreases with the increase of distance between the object and the tracker due to the increase of positional displacement (Equation 1).
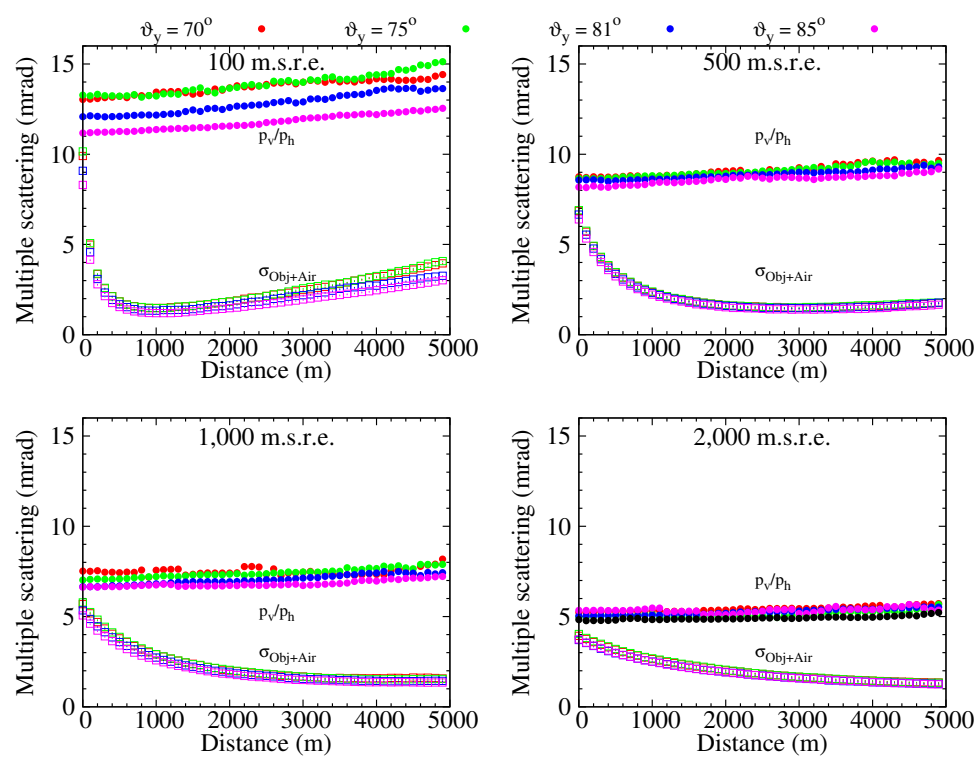

Figure 4: The ratio of momentum directions (filled circles) and the multiple scattering (empty rectangles) are shown as a function of distance from the object for 100, 500, 1,000, and 2,000 m.s.r.e. thick objects [5].

\section{Improvement of muon imaging by the measurement of low-energy muon spectra}

The muographic imaging of few-meter-thick objects requires the extension and improvement of the zenith-angle and altitude dependent low-energy muon spectra data sets. For this aim, we have designed a rotatable muon spectrometer (Figure 5) to quantify the muon spectra by the precise measurement the absorption and multiple scattering of muons. Our spectrometer is designed to be 
a consecutive series of nineteen MWPC with a position resolution of approx. $3.5 \mathrm{~mm}$ and variable number of lead plates with variable thickness. Actually, the spectrometer consists of nine $40 \mathrm{~cm}$ by $40 \mathrm{~cm}$ sized MWPCs and eight 20-cm-thick lead absorbers. The spectrometer has the same data acquisition and detector control system as the MMOS applied for volcano monitoring.
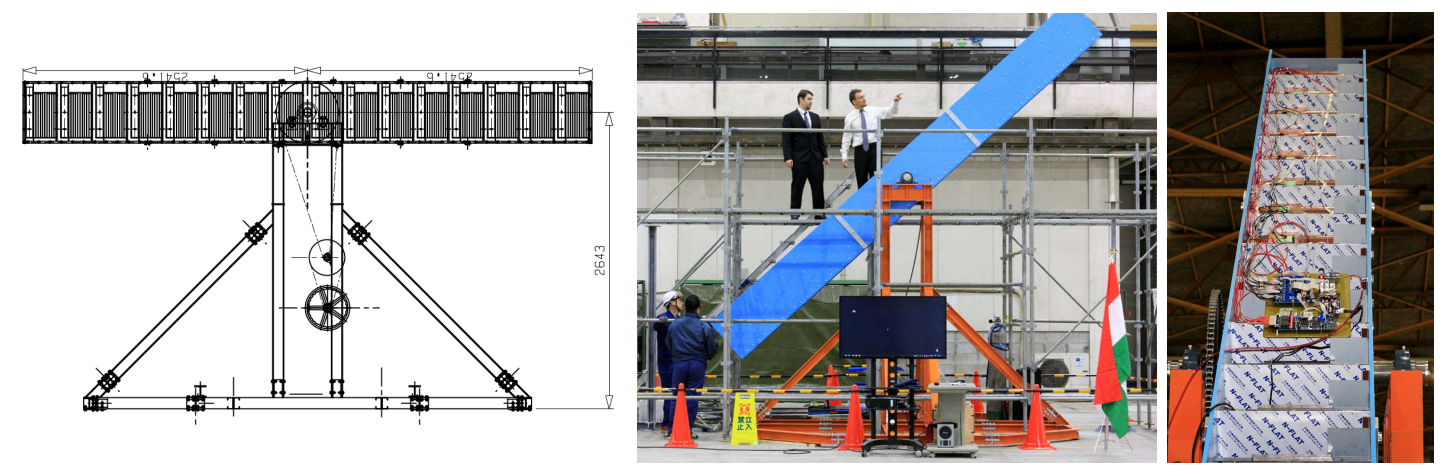

Figure 5: A schematic drawing of the spectrometer consists of 19 MWPCs and 18 lead absorbers (left). A photograph of the rotatable spectrometer (middle). A photograph of the spectrometer with nine MWPCs from front view (right).

The first data sets were being recorded by the first prototype since January 2019 to optimize the structure of the spectrometer. The energy loss and scattering effects across the detector structure were quantified with GEANT4 simulation [17]. The detector structure and materials (1-cm-thick iron support structure, 1-cm-thick Al absorber support structures and the lead absorbers) were implemented. Muons with uniformly distributed energies between 0 and $20 \mathrm{GeV}$ were injected across the spectrometer. The standard electromagnetic interaction package of GEANT4 were applied to simulate the interaction of the muons with the detector materials. The muons and secondary particles were followed across the spectrometer and their energies, position coordinates and momentum directions were recorded on event-by-event as ASCII files.

In the next stage of the study, the simulation data set were analyzed on event-by-event base. The distribution of muons that could reach a certain detector were determined. The average values and root-mean-square values of the distributions were calculated to quantify the range of muons across the spectrometer. Across the first prototype, the muon energies ranged up to $2.5 \mathrm{GeV}$ (left of Figure 6). Here, one absorber can be approximated to 20 -cm-lead equivalent. We note that the multiple scattering of muons contribute also to the loss of particles besides the energy loss processes, and this scattering effect was increased from 0 to $25 \%$ with the increase of absorbers (left panel of Figure 6).

The measured data were also analyzed and the flux of muons were determined after each absorber by correction on detector acceptance, dead time, tracking efficiency. For fluxes calculation, those tracks were chosen that penetrated across at least three detectors (two absorbers) and their goodness of fit $\left(\chi^{2} / n d f\right)$ were found to below 20 . The measured fluxes measured after 2, 3, 4, 5, 6,7 , and 8 absorbers are shown as a function of the simulated energies in right panel of Figure 6. The measured fluxes show good agreement with a global fit of earlier experimental data [18].

The construction of the spectrometer is expected to be completed in August 2019. The complete spectrometer is expected to measured the spectrum up to the energy of about $5 \mathrm{GeV}$. The 
extend the energy scale below $0.5 \mathrm{GeV}$ we are investigating the possibility to reduce the amount of absorbers to approximately 100 radiation length and simultaneously measure the absorption and multiple scattering of muons. The application of scintillators as time-of-flight modules is also under consideration for this aim and for the separation of horizontally arriving muons.

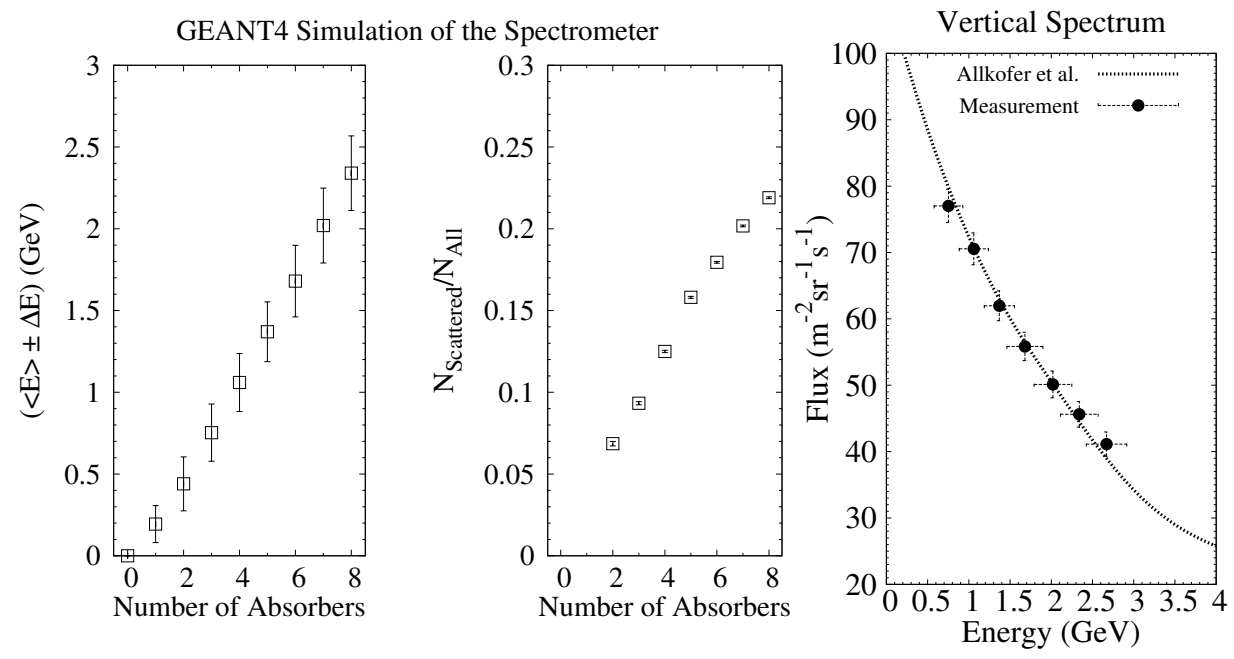

Figure 6: The simulated muon energies after 20-cm-thick lead absorbers in the spectrometer (left). The ratio of out-scattered muons to the expected number of muons after the absorbers. (middle). Our first measured vertical spectrum shows a agreement with an earlier proposed fit [18] (right).

\section{Summary and discussion}

We have reviewed various applications of MMOS technology for high-definition muon imaging, investigated the limits and feasibilities of muography, and reported the status of an ongoing $\mathrm{R} \& \mathrm{D}$ work for the measurement of low-energy muon spectra. Our technology provide a reasonable angular resolution of few mrad, its size can be safely optimized from a few centimeters to a few tens of meters for the desired application, such as for inspecting the structure of human made constructions or imaging active volcanoes [19]. Although, MMOS can be applied to directional sensitive measurement of cosmic-rays showers [20], its further optimization, such as improvement of gas supply, will be required to use this technology in large-scale experiments.

\section{Acknowledgement}

The technical support provided by the REGARD is gratefully acknowledged. We acknowledge the support of the Ministry of Education, Culture, Sports, Science and Technology, Japan Integrated Program for the Next Generation Volcano Research and Human Resource Development and Hungarian-Japanese Bilateral Research Fund For Science and Technology (TÉT_16-1-2016-0198). 


\section{References}

[1] L. W. Alvarez et al.: Search for hidden chambers in the pyramids, Science 167 (1970) 832-839

[2] H. K. M. Tanaka et al.: High resolution imaging in the inhomogeneous crust with cosmic-ray muon radiography: The density structure below the volcanic crater floor of Mt. Asama, Japan, Earth and Planetary Science Letters 263 (2007) 104-113

[3] H. K. M. Tanaka et al.: Radiographic visualization of magma dynamics in an erupting volcano, Nat. Commun. 5 (2014) 3381

[4] L. Oláh et al.: High-definition and low-noise muography of the Sakurajima volcano with gaseous tracking detectors, Scientific Reports 8 (2018) 3207

[5] L. Oláh et al.: Investigation of the limits of high-definition muography for observation of Mt Sakurajima, Phil. Trans. R. Soc. A 377 (2018) 20180135

[6] D. Varga et al.: Detector developments for high performance Muography applications, Nuclear Instruments and Methods in Physics Research Section A https://doi.org/10.1016/j.nima.2019.05.077

[7] L. Oláh et al.: Muographic Observation of Density Variations in the Vicinity of Minami-Dake Crater of Sakurajima Volcano, Journal of Disaster Research 14 (2019) Dr14-5-9926

[8] D. Varga et al.: High Efficiency Gaseous Tracking Detector for Cosmic Muon Radiography, Advances in High Energy Physics 2016 (2016) 1962317

[9] Online monitoring page of MMOS: https://mmos.muographers.org/

[10] L. Oláh et al.: The first prototype of an MWPC-based borehole-detector and its application for muography of an underground pillar, Geophysical Exploration 71 (2018) 61-68

[11] D. Varga et al.: Close cathode chamber: Low material budget MWPC, Nuclear Instruments and Methods in Physics Research Section A 698 (2013) 11-18

[12] L. Oláh et al.: CCC-based muon telescope for examination of natural caves, Geosci. Instrum. Method. Data Syst. 1 (2012) 229-234

[13] M. Y. Guan et al.: A parametrization of the cosmic-ray muon flux at sea-level (2015) arXiv:1509.06176.

[14] J. W. Keuffel JW, et al.: Zenith-angle distribution of ultra-high energy muons, Proc. 11th Int. Conf. on Cosmic Rays 29 (1969)

[15] H. E. Bergenson et al.: Zenith-angle dependence of cosmic-ray muons, Phys. Rev. Lett. 27 (1971)

[16] L. Bergamasco et al.: Muon sea-level intensity and primary cosmic-ray nucleon spectrum in the (1-100) TeV energy range from the Mt. Blanc underground experiment, Il Nou. Cim. C 6 (1983)

[17] S. Agostinelli et al:: Geant4 - a simulation toolkit, Nucl. Instrum. Meth. A 506 (2003) 250-303

[18] O. C. Allkofer et al.: The absolute cosmic-ray muon spectrum at sea-level Physics Letters 36B (1971) 425-427

[19] Webpage of Muographic Observation Instrument: http://www.eu-jp-tthelpdesk.eu/technologies/muographic-observation-instrument/

[20] L. Oláh and D. Varga: Investigation of soft component in cosmic ray detection, Astroparticle Physics 93 (2017) 17-27s 